\title{
Investigations of Three-Dimensional Shock/Shock Interactions over Symmetrical Intersecting Wedges
}

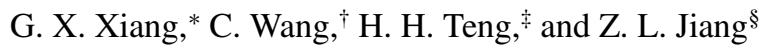 \\ Chinese Academy of Sciences, 100190 Beijing, People's Republic of China
}

\begin{abstract}
DOI: $\underline{10.2514 / 1 . J 054672}$
This study explored inviscid supersonic corner flows induced by three-dimensional symmetrical intersecting compression wedges by introducing the spatial dimension reduction theoretical approach to transform the threedimensional steady shock/shock interaction problem into a two-dimensional pseudosteady problem; this method allows not only wave configurations, which include regular reflection and Mach reflection, to be determined accurately, but also flowfield characteristics, which include density, temperature, pressure, and total pressure recovery coefficient near the regular reflection point (or in the vicinity of the Mach reflection triple point), as well as the location and the strength of the Mach stem. Theoretical results were compared to numerical simulation (performed by solving three-dimensional inviscid Euler equations with an non-oscillatory and non-free-parameters dissipative finite difference scheme) and analyzed thoroughly. The effects of inflow Mach number, sweep angle, and wedge angle on flowfield parameters and wave configurations were also considered. The influence of sweep angle is negligible, but the effects of Mach number and wedge angle are significant.
\end{abstract}

\begin{tabular}{|c|c|c|}
\hline \multicolumn{3}{|r|}{ Nomenclature } \\
\hline$i$ & $=$ & incident wave \\
\hline$K$ & $=$ & kink \\
\hline$M_{m}$ & $=$ & Mach number of Mach stem \\
\hline$M_{s 1}, M_{s 2}$ & $=$ & components of inflow Mach number vector \\
\hline$M_{0}$ & $=$ & inflow Mach number \\
\hline$m$ & $=$ & Mach stem \\
\hline$m^{\prime}$ & $=$ & secondary Mach stem \\
\hline$R$ & $=$ & reflection point \\
\hline$r$ & $=$ & reflected wave \\
\hline$r^{\prime}$ & $=$ & secondary reflected wave \\
\hline$s$ & $=$ & slipstream \\
\hline$s^{\prime}$ & $=$ & secondary slipstream \\
\hline$T$ & $=$ & first triple point \\
\hline$T^{\prime}$ & $=$ & secondary triple point \\
\hline$\beta$ & $=$ & shock angle in the $x$ direction \\
\hline$\beta_{n}$ & $=$ & $\begin{array}{l}\text { shock angle perpendicular to leading edge } \boldsymbol{O A} \text { and } \\
\boldsymbol{O C}\end{array}$ \\
\hline$\theta$ & $=$ & wedge angle \\
\hline$\theta_{v}$ & $=$ & angle between virtual wall and horizontal line \\
\hline$\lambda$ & $=$ & sweep angle \\
\hline$\nu$ & $=$ & dihedral angle \\
\hline$\chi$ & $=$ & first triple point trajectory angle \\
\hline$\chi^{\prime}$ & $=$ & second triple point trajectory angle \\
\hline 1 & $=$ & incident waves \\
\hline 2 & $=$ & reflected waves \\
\hline 3 & $=$ & Mach stem \\
\hline 4 & $=$ & slip lines \\
\hline 5 & $=$ & spiral vortices \\
\hline 6 & $=$ & secondary reflected waves \\
\hline
\end{tabular}

Received 11 August 2015; revision received 10 November 2015; accepted for publication 14 November 2015; published online 11 January 2016. Copyright $\odot 2015$ by the American Institute of Aeronautics and Astronautics, Inc. All rights reserved. Copies of this paper may be made for personal or internal use, on condition that the copier pay the $\$ 10.00$ per-copy fee to the Copyright Clearance Center, Inc., 222 Rosewood Drive, Danvers, MA 01923; include the code 1533-385X/15 and \$10.00 in correspondence with the CCC.

*Doctor, State Key Laboratory of High-Temperature Gas Dynamics, Institute of Mechanics.

${ }^{\dagger}$ Associate Professor, State Key Laboratory of High-Temperature Gas Dynamics, Institute of Mechanics; wangchun@imech.ac.cn (Corresponding Author).

${ }^{\ddagger}$ Associate Professor, State Key Laboratory of High-Temperature Gas Dynamics, Institute of Mechanics.

${ }^{\S}$ Professor, State Key Laboratory of High-Temperature Gas Dynamics, Institute of Mechanics. Associate Fellow AIAA. $\begin{array}{lll}7 & = & \text { contact discontinuity } \\ 8 & = & \text { near-wall Mach shock }\end{array}$

\section{Introduction}

T $\mathrm{N}$ AEROSPACE engineering, the effective design of modern supersonic and hypersonic vehicles requires thorough understanding of the physical flowfield structure of corner flows. Corner flows exist in fuselage wing junctions, rectangular inlet diffusers and ducts, the intersection of several control surfaces, and turbomachine blade-hub junctions; and three-dimensional shock/shock interaction (SSI) often occurs in corner flows. This interaction produces complex three-dimensional (3-D) shock-wave configurations, which lead to total pressure loss. There already exists a wealth of research regarding two-dimensional (2-D) shock reflection and SSI theories.

The earliest research on shock-wave reflection can be traced back to 1878, when Mach first observed and recorded reflection phenomena of shock waves and discovered two distinct shock-wave reflection configurations: regular reflection (RR) and Mach reflection (MR) [1]. Von Neumann built analytical approaches for describing RR and the MR wave configurations called two-shock theory for RR and threeshock theory for MR, respectively [2,3]. Kawamura developed the $(p, \theta)$ polar method ( $p$ is the flow static pressure, and $\theta$ is the flow deflection angles), which has proven a highly effective tool for analyzing shock reflection and interaction [].

Ben-Dor systematically summarized the progress in 2-D shockwave reflection phenomena and related analytical theory, including both steady shock reflection and pseudosteady shock reflection [5]. According to his research, possible shock reflection configurations of 2-D symmetric pseudosteady flows include regular reflection (RR) and the direct-Mach reflection (DiMR) [6] (Fig. 1). DiMR can be further divided into three types: single-Mach reflection (SMR); transitional-Mach reflection (TMR), discovered by Smith [7]; and double-Mach reflection (DMR) (Figs. 1b-1d). DMR can be further divided into two types, depending on whether the trajectory angle of the first triple point is larger or smaller than that of the second triple point: positive-double Mach reflection (DMR+, $\left.\chi>\chi^{\prime}\right)$, and negative double-Mach reflection (DMR-, $\chi<\chi^{\prime}$ ); these two types of DMR were studied by Ben-Dor [8] as well and were named as such by Lee and Glass [9].

As opposed to well-established theory regarding the 2-D shock reflection problem, there has been little established theoretical work on 3-D shock reflection and SSI problems, likely due to the complexity of flow features of 3-D SSI, though 3-D is much more common in practice than 2-D shock flow. Charwat and Redekeopp studied 3-D shock-wave interactions as early as 1967, observing entropy waves (slip lines) and reflected waves in experiments [10]. 


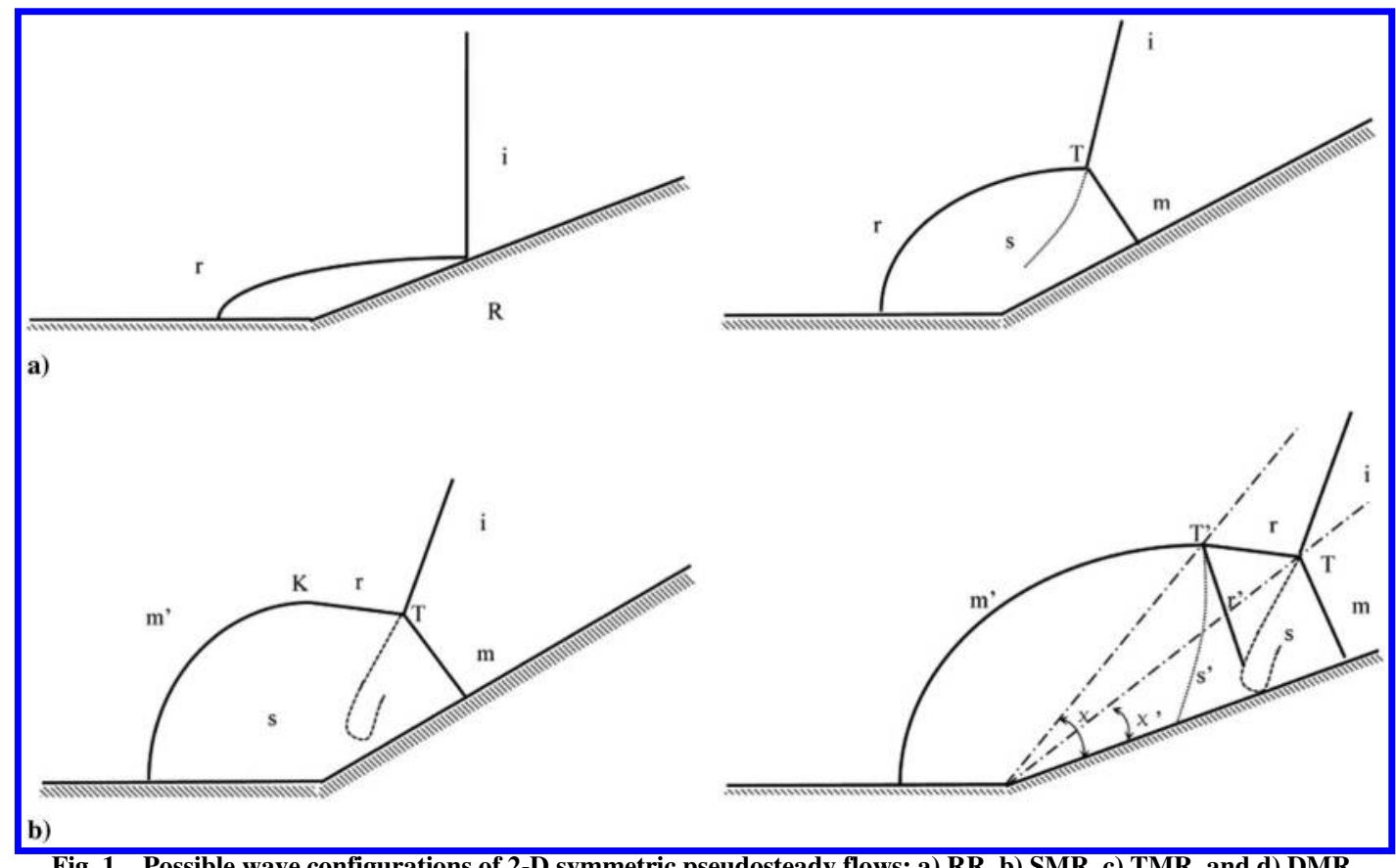

Fig. 1 Possible wave configurations of 2-D symmetric pseudosteady flows: a) RR, b) SMR, c) TMR, and d) DMR.

Watson and Weinstein noted that the flowfield appears to be basically conical, except for cases of serious viscous interaction effects [11]. Marconi used a second-order, finite difference marching technique to predict the inviscid supersonic/hypersonic flowfield of conical internal corners [12], and several numerical and analytical studies on corner flows formed by intersecting wedges have been conducted by Goonko et al., who discussed the effects of angles of inclination, sweep angles of the leading edges, dihedral angles, and corner ribs $[13,14]$. Skews et al. [15], Naidoo [16], and Naidoo and Skews [17] conducted extensive research on corner flows and discovered that the flow is no longer self-similar if the sharp corner is replaced by a camber.

Most of the previous research on 3-D shock reflection and SSI were conducted experimentally and numerically; analytical study, however, is generally lacking. In the present study, a classical method called spatial dimension reduction was applied to 3-D SSI, and numerical simulations were conducted to validate the analytical results. The theoretical solutions agreed well with the numerical results.

The configurations of 3-D SSI in supersonic 3-D steady flows are studied both analytically and numerically in this study. The spatial dimension reduction approach and the numerical methods used here are presented in detail in Sec. II. In Sec. III, the theoretical solution to the corner flow near the reflection point (or in the vicinity of the triple point) is obtained using the proposed method, and key parameters such as inflow Mach number, sweep angle, and wedge angle, plus their effects on the flowfield and wave configuration, are discussed analytically and numerically. Section IV provides a brief summary and conclusions.

\section{Spatial Dimension Reduction Approach and Numerical Methods}

The simplest 3-D steady SSI is induced by two intersecting wedges in a supersonic flow, which can be found in the threedimensional hypersonic inlet of a scramjet engine. As shown in Figs. 2 and 3 , there are two symmetrical intersecting wedges with specific geometric angles, and the angle between the two bottom planes of the wedges is defined as $\nu$. The two shock planes are induced by the bottom wedge and the lateral wedge with sweep angle $\lambda$ and wedge angle $\theta$.

\section{A. Spatial Dimension Reduction Approach}

For the 3-D SSI induced by the two intersecting wedges, numerical simulations indicate that, in the direction of the intersecting line of two planar shocks, the flow feature is self-similar, for either regular interaction (RI) or Mach interaction (MI) (Fig. 2) [13,14]. The basic idea of spatial dimension reduction is that 3 -D steady SSI can be transformed into a 2-D moving SSI problem with time evolution by reducing one characteristic spatial dimension to a temporal dimension.

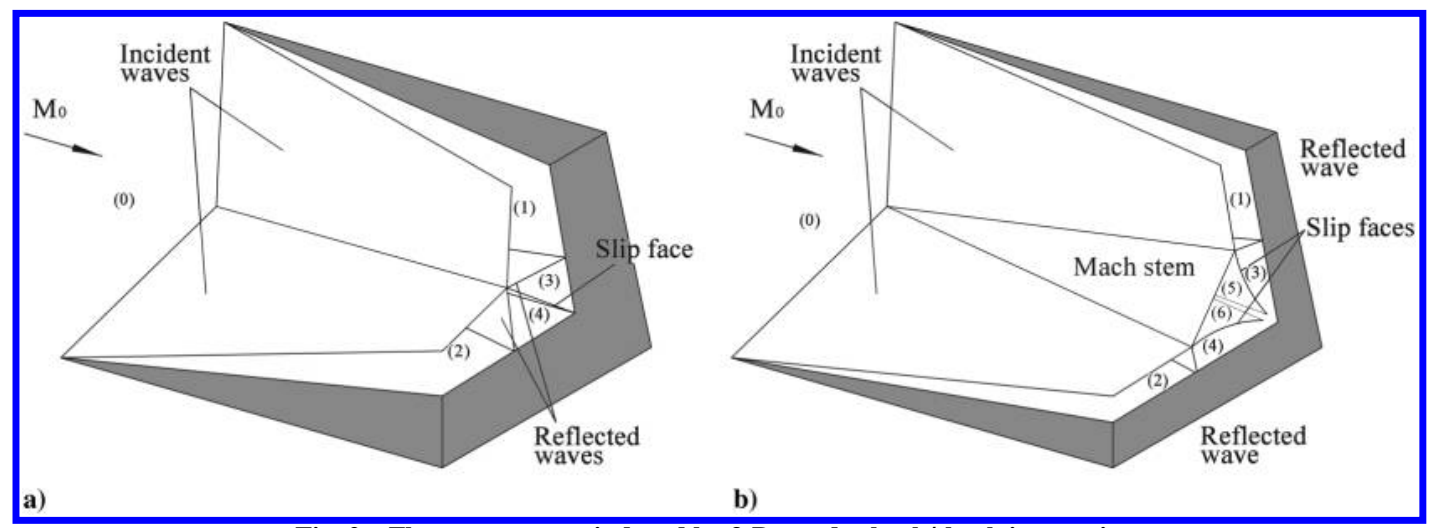

Fig. 2 Flow parameters induced by 3-D steady shock/shock interaction. 


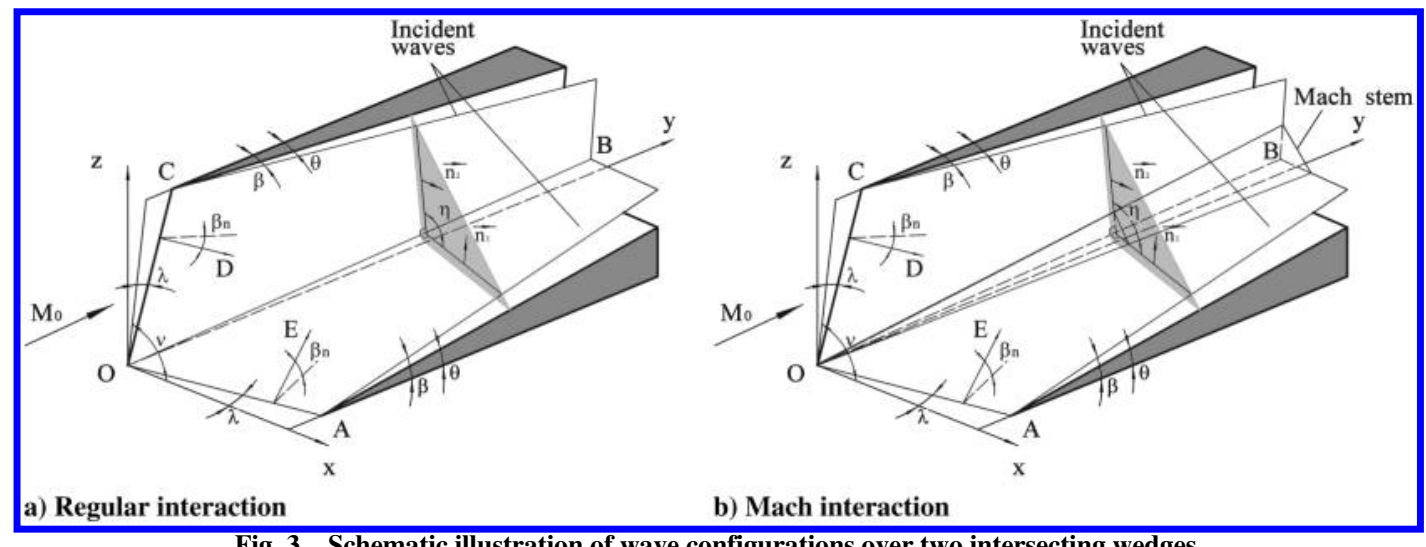

Fig. 3 Schematic illustration of wave configurations over two intersecting wedges.

As shown in Fig. $\underline{3 a}$ and $\underline{3 b}$, the direction of the interacting line of two shock planes $\boldsymbol{O} \overline{\boldsymbol{B}}$ is defined as the characteristic direction, and the planes perpendicular to $\boldsymbol{O B}$ as characteristic planes. The velocities in the flowfield can be decomposed into two respective components in the characteristic direction and in the characteristic planes. According to the properties of oblique shock waves (and strict theoretical analysis thereof), the velocity component in the characteristic direction remains constant in the entire flowfield, which can be dissociated from other velocity components or theoretical analyses. Thus, the spatial dimension in the characteristic direction can be considered a temporal dimension; the distance in the characteristic direction can be treated as the product of time variation and the constant characteristic velocity. The flowfields in different characteristic planes along the characteristic direction can then be considered the movement of two-dimensional shock waves that develops with time evolution, if the characteristic planes are projected to the characteristic plane passing through the original point $O$ as shown in Fig. 2.

The spatial dimension reduction approach is applicable for RI and MI (Figs. $2 \underline{\mathrm{a}}, \underline{2 \mathrm{~b}}, \underline{3 \mathrm{a}}$ and $\underline{3 \mathrm{~b}}$ ). $M_{S 1}$ and $M_{S 2}$ are the components of the inflow Mach number vector normal to shock $S_{1}$ and $S_{2}$, respectively, and can be calculated as follows:

$$
M_{S 1}=M_{S 2}=M_{0} \sin \beta_{n} \cos \lambda
$$

where $M_{0}$ is the inflow Mach number, and $\beta_{n}$ represents the angle of shock planes perpendicular to leading edges $\boldsymbol{O A}$ and $\boldsymbol{O C}$.

The vector $\boldsymbol{O B}$ and angle $\eta$ are obtained as follows:

$$
\boldsymbol{O B}=\boldsymbol{n}_{2} \times \boldsymbol{n}_{1}
$$

$$
\cos \eta=\cos \left(\boldsymbol{n}_{2}, \boldsymbol{n}_{1}\right)=\cos (\boldsymbol{O} \boldsymbol{A} \times \boldsymbol{E}, \boldsymbol{D} \times \boldsymbol{O C})
$$

where $\boldsymbol{E}$ is the vector in shock plane $S_{1}$ normal to $\boldsymbol{O A}$, and $\boldsymbol{D}$ is the vector in shock plane $S_{2}$ normal to $\boldsymbol{O C}$.
The wave configurations of transformed 2-D SSI can then be determined by shock-polar analysis (see Fig. 4a) because a problem of 2-D moving SSI can be solved analytically [18]. Generally, the type of RI must first be defined using the dimension reduction approach; for the RI configuration, the trajectory of the interaction point is defined here as a virtual wall, and the moving reference frame is set on the intersection point. The problem can then be transformed into a 2-D steady shock-wave interaction problem, as shown in Fig. $4 \mathrm{~b}$.

The angle of the virtual wall $\theta_{\nu}$ and the angles between shock waves and virtual walls $\alpha_{1}$ and $\alpha_{2}$ can be calculated as follows:

$$
\begin{aligned}
& \theta_{\nu}=\frac{\pi}{2}-\arctan \left(\frac{M_{S 2} \sin \eta}{M_{S 1}+M_{S 2} \cos \eta}\right) \\
& \alpha_{1}=\eta-\frac{\pi}{2}+\theta_{\eta}, \quad \alpha_{2}=\frac{\pi}{2}-\theta_{\eta}
\end{aligned}
$$

Figure 5a shows a schematic illustration of the $p, \theta$ polar solution near the RI reflected point. After the compression of incident waves $I_{1}$ and $I_{2}$, the flow state is raised from state 0 to states 1 and 2, respectively. The reflected shock polars $R_{1}$ and $R_{2}$ interact at two points, where the intersecting points indicate that pressure and angle conditions are matched behind two reflected waves, which are divided by a slip line (Fig. $\underline{4 b}$ ). If the principle of minimal entropy generation is assumed, a lower point with lower pressure is possible; therefore, all the parameters in all regions can be solved theoretically.

As shown later, an example of numerical simulation was made to validate the theoretical results. Parameters were selected as $M_{0}=5$, $\nu=90 \mathrm{deg}, \lambda=3 \mathrm{deg}$, and $\theta=3 \mathrm{deg}$. The theoretical analysis in Fig. $3 \mathrm{a}$ agrees well with the numerical results shown in Fig. $5 \mathrm{~b}$.

If the two reflected shock waves $R_{1}$ and $R_{2}$ do not intersect but do intersect with $I_{1}$ and $I_{2}$, respectively, an MI will appear (Fig. $\underline{6 a}$ ). A

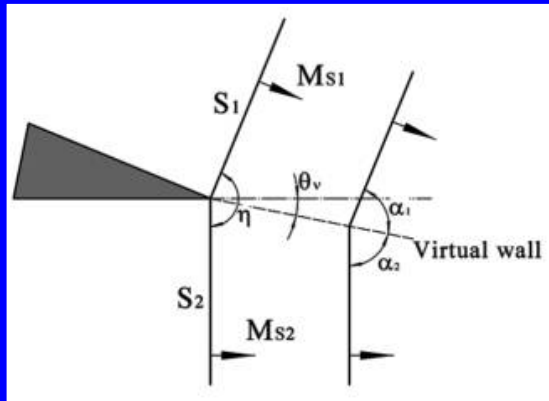

a) RI of 2-D moving shock waves

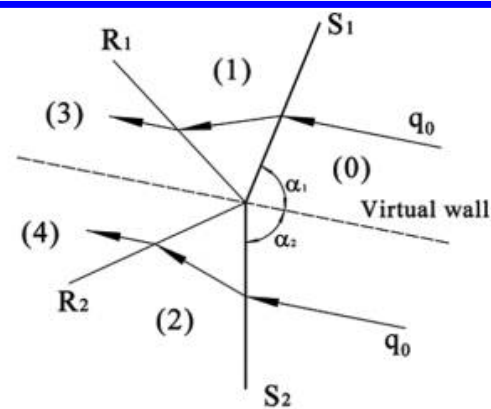

b) 2-D steady shock interaction 
schematic illustration of an MI is shown in Fig. 7a, where a Mach stem grows with the propagation of two moving shock waves. Because the solution to an MI is more difficult than one for an RI, the problem cannot be solved using a single reference frame transformation. One effective approach is to find a virtual wall in the flowfield and solve the problem of shock reflection on both sides of the virtual wall. The virtual wall is defined as a line perpendicular to the Mach stem; then, frames of reference can be attached to triple points $T_{1}$ and $T_{2}$, respectively (Fig. 7b).

A simple solution to the problem of 2-D moving SSI can be established based on shock dynamics. The Mach stem strength $M_{m}$ and angle $\theta_{\nu}$ between the virtual wall and the horizontal line can be acquired by solving the problem of Mach reflection at both sides of the virtual wall $[18,19]$.

$M_{m}$ and $\theta_{\nu}$ can be calculated with the following equations [18,20]:

$$
\tan \theta_{\nu}=\left(\frac{M_{m}}{M_{s 2}}\right) \frac{\left[1-\left(M_{s 2} / M_{m}\right)^{2}\right]^{1 / 2}\left\{1-\left[f\left(M_{m}\right) / f\left(M_{s 2}\right)\right]^{1 / 2}\right\}}{1+\left[f\left(M_{m}\right) M_{m} / f\left(M_{s 2}\right) M_{s 2}\right]}
$$

and

$$
\begin{aligned}
& \tan \left(\pi-\eta-\theta_{\nu}\right) \\
& \quad=\left(\frac{M_{m}}{M_{s 1}}\right) \frac{\left[1-\left(M_{s 1} / M_{m}\right)^{2}\right]^{1 / 2}\left\{1-\left[f\left(M_{m}\right) / f\left(M_{s 1}\right)\right]^{1 / 2}\right\}}{1+\left[f\left(M_{m}\right) M_{m} / f\left(M_{s 1}\right) M_{s 1}\right]}
\end{aligned}
$$

where

$$
f(M)=\exp \left[-\int \frac{2 M d M}{\left(M^{2}-1\right) K(M)}\right]
$$

Here, $K(M)$ is a slowly varying function expressed as follows:

$$
K(M) 2\left(2 \mu+1+M^{-2}\right)^{-1}\left(1+\frac{2}{\gamma+1} \frac{1-\mu^{2}}{\mu}\right)^{-1}
$$

where

$$
\mu=\left[\frac{(\gamma-1) M^{2}+2}{2 \gamma M^{2}-(\gamma-1)}\right]
$$

represents the Mach number for the propagation of a moving shock relative to the flowfield behind it.
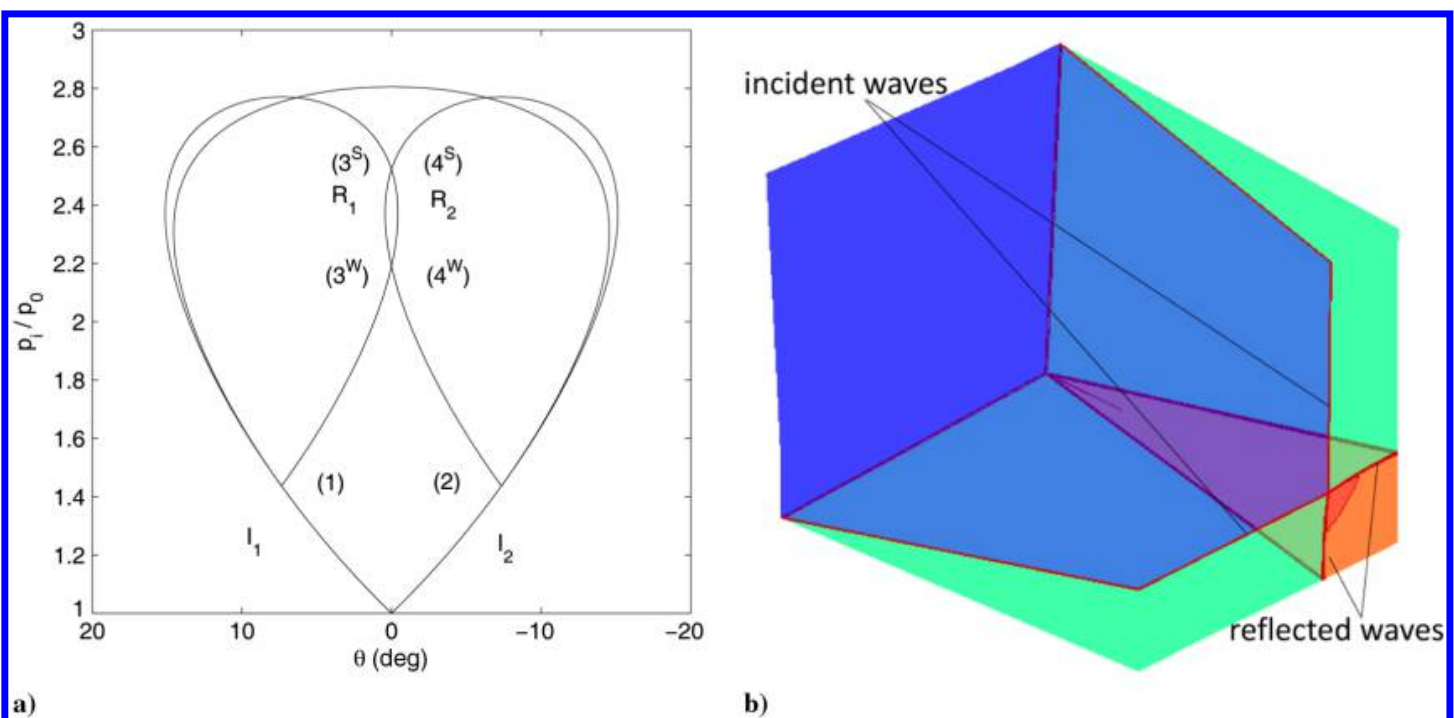

Fig. 5 Shock polars of RI configuration and numerical validation: a) $(p, \theta)$ polars of $\mathrm{RI}$, and b) numerical simulation example.

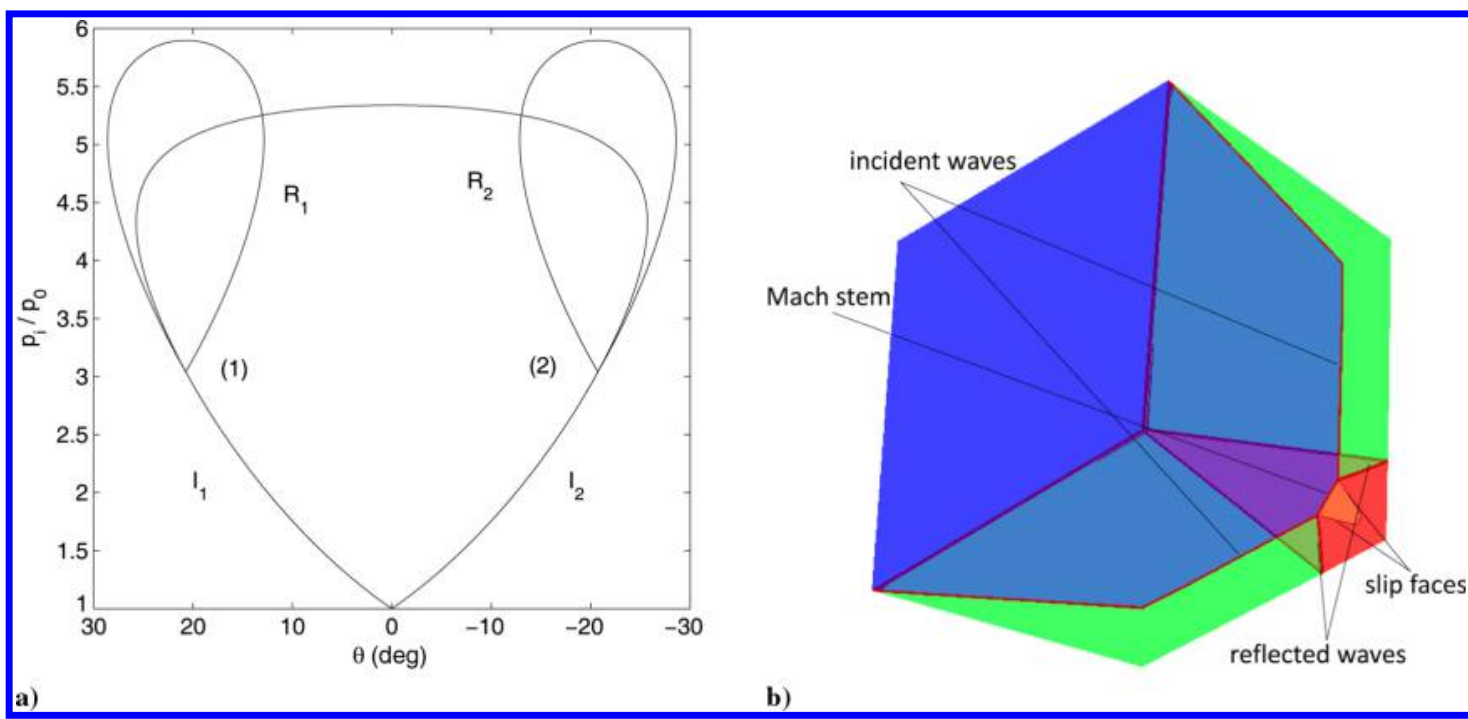

Fig. 6 Shock polars of MI configuration and numerical validation: a) $(p, \theta)$ polars for MI, and b) numerical simulation example. 
The angles between the triple-point trajectories and the virtual walls $\gamma_{1}$ and $\gamma_{2}$ are expressed as follows:

$$
\begin{gathered}
\gamma_{1}=\arctan \left(\frac{M_{m}+\sec \left(\eta+\theta_{\nu}\right) M_{s 2}}{-\tan \left(\eta+\theta_{\nu}\right) M_{m}}\right) \\
\gamma_{2}=\arctan \left(\frac{M_{m}-\sec \theta_{\nu} M_{s 2}}{\tan \theta_{\nu} M_{m}}\right)
\end{gathered}
$$

A numerical example of a 3-D steady MI is presented in Fig. 7b. The selected parameters are $M_{0}=5, \nu=90 \mathrm{deg}, \lambda=5 \mathrm{deg}$, and $\theta=10 \mathrm{deg}$, and the numerical result is shown in Fig. 5b. The 3-D shock interaction configuration is MI, consistent with the theoretical analysis (Fig. 5a).

Finally, the values of all zones should be correlated to the 3-D steady flow (Fig. 2). State parameters, such as temperature, pressure, density, and total pressure recovery coefficient, are identical to those of the 2-D pseudosteady flow. The velocities and Mach number, however, should be composed with the decomposed constant velocity and Mach number vectors obtained previously (Fig. 3). The corner flow properties of 3-D steady SSI can be analyzed theoretically using this procedure.

\section{B. Numerical Methods}

The viscosity effects are negligible in 3-D SSI, and so governing equations are simplified to three-dimensional Euler equations as follows:

$$
\frac{\partial U}{\partial t}+\frac{\partial F}{\partial x}+\frac{\partial G}{\partial y}+\frac{\partial H}{\partial z}=0
$$

where $U, F$, and $G$ denote the state variables and fluxes in the $x, y$, and $z$ directions, respectively:

$$
\begin{aligned}
U & =\left(\begin{array}{c}
\rho \\
\rho u \\
\rho v \\
\rho w \\
\rho E
\end{array}\right), \quad F=\left(\begin{array}{c}
\rho u \\
\rho u^{2}+p \\
\rho u v \\
\rho u w \\
(\rho E+p) u
\end{array}\right), \quad G=\left(\begin{array}{c}
\rho v \\
\rho u v \\
\rho v^{2}+p \\
\rho v w \\
(\rho E+p) v
\end{array}\right), \\
H & =\left(\begin{array}{c}
\rho w \\
\rho u w \\
\rho v w \\
(\rho E+p) w
\end{array}\right)
\end{aligned}
$$

For the perfect gas, $E$ represents the total internal energy per unit mass:

$$
E=\frac{p}{(\gamma-1) \rho}+\frac{1}{2}\left(u^{2}+v^{2}+w^{2}\right)
$$

The discretization of the governing equations is

$$
\begin{aligned}
& \left(\frac{\partial U}{\partial t}\right)_{i, j, k}^{n}+\frac{1}{\Delta x}\left(F_{i+\frac{1}{2}, j, k}^{n}-F_{i-\frac{1}{2}, j, k}^{n}\right)+\frac{1}{\Delta y}\left(G_{i, j+\frac{1}{2}, k}^{n}-G_{i, j-\frac{1}{2}, k}^{n}\right) \\
& +\frac{1}{\Delta z}\left(H_{i, j, k+\frac{1}{2}}^{n}-H_{i, j, k-\frac{1}{2}}^{n}\right)=0
\end{aligned}
$$

where the second-order NND scheme is applied for the convective terms $F, G$, and $H$, and the minmod function is the limiter. The computational mesh uses the orthogonalized uniform structured mesh. Because the mesh quantity is excessively large with a grid system of $200 \times 200 \times 120$, Message Passing Interface (MPI) parallel programs were used in the code. Mesh independence tests were performed to ensure that all the results produced are independent of the type of mesh chosen for numerical simulations. The inlet of the computational zone is given by fixed inflow, the farfield is a nonreflecting boundary, and the walls are solid slipping. Computations were conducted on an eight-core computer (Dell Inc.).

\section{Results and Discussion}

Figure 8 presents the comparison between analytical results and numerical results as well as experimental results of West and Korkegi

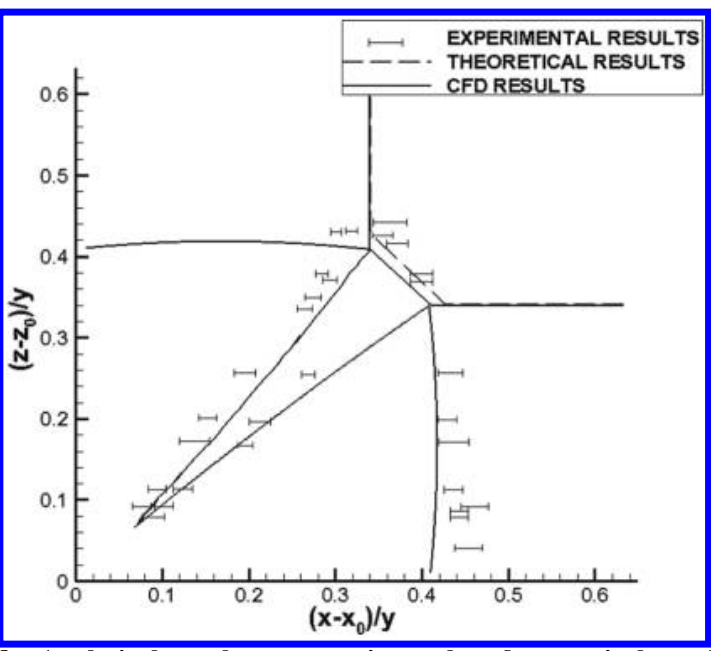

Fig. 8 Analytical results vs experimental and numerical results of sectional flowfield $\left(M_{0}=3, \nu=90 \mathrm{deg}, \lambda=0 \mathrm{deg}, \theta=9.5 \mathrm{deg}\right)$.

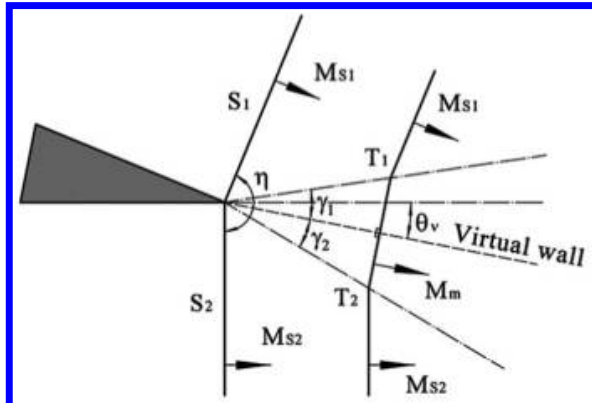

a) MI of 2-D moving shock waves

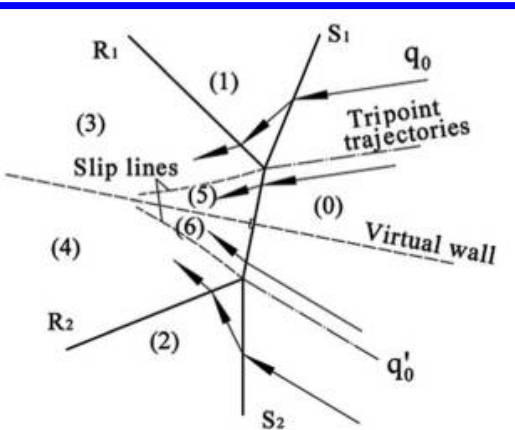

b) 2-D pseudosteady shock/shock interaction 

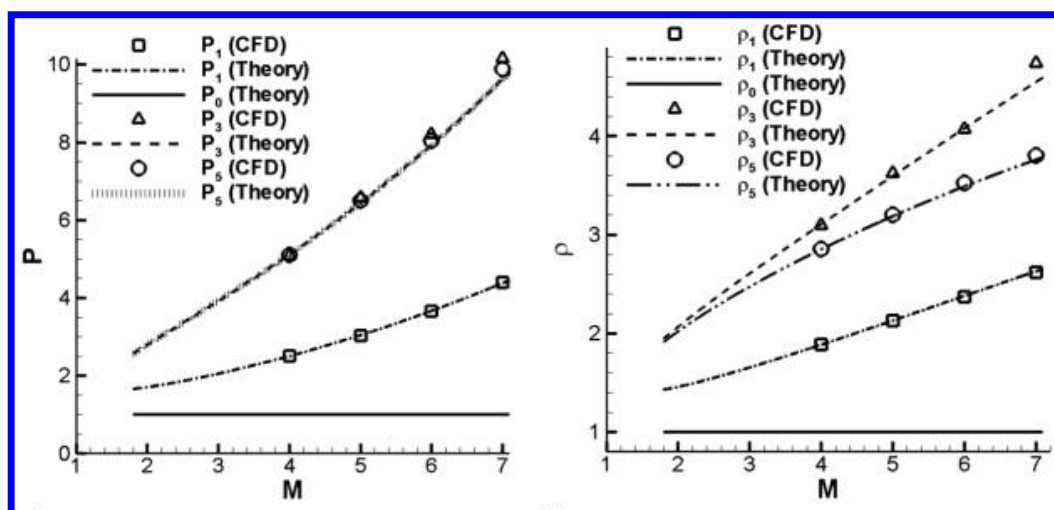

a)

b)
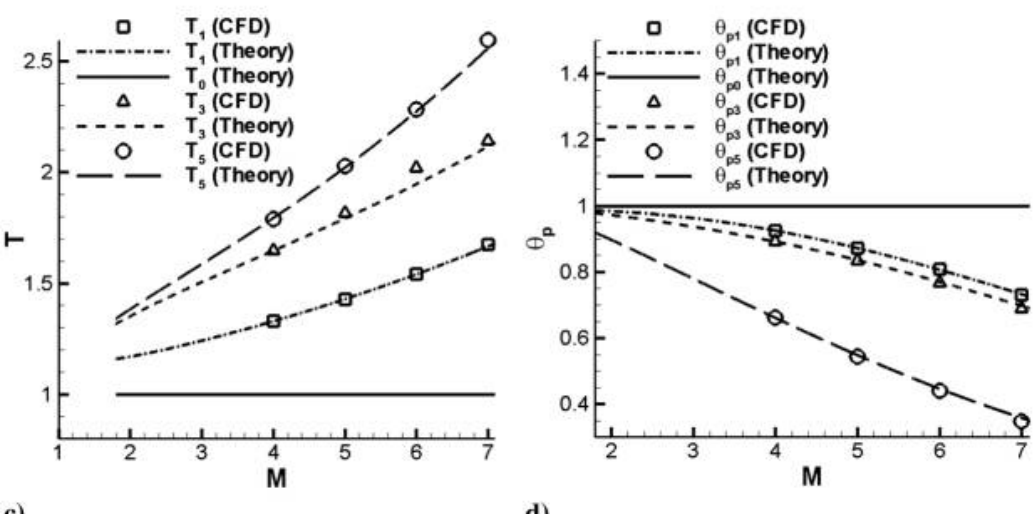

d)

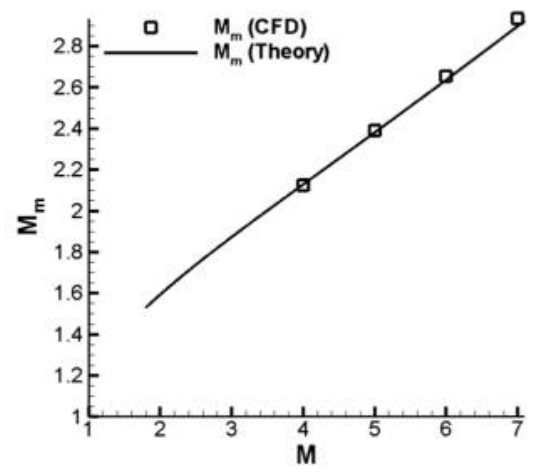

e)

Fig. 9 Parameter variations of flowfield with inflow Mach number: a) pressure, b) density, c) temperature, d) total pressure recovery coefficient, and e) Mach stem strength.

in 1972 [21]. The coordinates $x$ and $z$ are scaled with $y$ so that they become conical, self-similar variables. $z_{0}$ and $x_{0}$ represent the distances of the bottom wedge and the top wedge. The error bars are experimental results of West and Korkegi in 1972. It can be seen from the figure that the theoretical results of the location of incident waves and Mach stem agree well with the experimental and numerical results. CFD is the abbreviation of Computational Fluid Dynamics. For the strength of Mach stem, the theoretical solution is $M_{m}=1.84$, and the numerical solution is about $M_{m}=1.82$.

In this section, key parameters' effects on the flowfield and wave configurations will be discussed analytically and numerically.

\section{A. Inflow Mach Number Effects}

The effects of the Mach number are illustrated here using examples of a corner flow with parameters $M_{0}=3 \sim 7, \nu=90$ deg, $\lambda=0 \mathrm{deg}$, and $\theta=10 \mathrm{deg}$. Shock-polar analysis on cross sections implies that the wave configurations at $M_{0} \leq 10, \nu=90 \mathrm{deg}$, $\lambda=0 \mathrm{deg}$, and $\theta=10 \mathrm{deg}$ cannot be an RI because the shock polars of reflected shock waves never intersect each other, in accordance with numerical simulation results. For cases with smaller Mach numbers, wave configurations are characterized by weak shock interaction, which is similar to the von Neumann reflection of weak shock waves in two-dimensional pseudosteady flows.

Figure $9 \mathrm{a}-9 \mathrm{c}$ shows the pressure, density, and temperature in zones 1, 3, and 5 with different inflow Mach numbers. As shown in Fig. 9 , state 0 represents dimensionless units of inflow pressure, density, and temperature. As the inflow Mach number $M_{0}$ increases, pressure, density, and temperature of zones 1-5 and Mach stem strength increase monotonously, and the total pressure recovery coefficient decreases gradually (Fig. 9a-9e). Because zones 3 and 5 are only divided by slip faces, they have the same pressure (Fig. 9a). As indicated in Figs. 9b and 9c, the density behind the Mach stem is lower than that behind the reflected waves, whereas the temperature behind the Mach stem is higher than that behind the reflected shock waves. The total pressure loss is more serious behind the Mach stem than that behind the reflected shock waves (Fig. 9d) because the entropy production passing through the incident shock waves and reflected shock waves is smaller than that passing through the Mach stem. 
The numerical results of corner flows in Mach number range from $3 \leq M_{0}<6$ with $\nu=90 \mathrm{deg}, \lambda=0 \mathrm{deg}$, and $\theta=10 \mathrm{deg}$ showing that the wave configuration is a single Mach interaction (SMI). Clear reflected shock waves, Mach stem, and slip lines can be observed for SMI (Figs. 10a and 10b). When the inflow Mach number is raised to 6, a transitional Mach interaction (TMI) occurs. The contact discontinuity originating from the triple points rolls up into spiral vortexes, and the reflected waves near the wedge become more concave in shape (Fig. 10c). The bending of the reflected shock wave occurs due to the action of compression waves from the wall. When $M_{0}=7$, the reflected waves cause a near-wall shock wave and secondary reflected shock wave, and it forms a DMI (see Fig. 10d). If the Mach number is increased to 8 or much larger, the wave configurations change into multishock Mach interactions, and the near-wall flow becomes more complicated.

The trajectory of the three-shock points of the Mach stem can be solved using the spatial dimension reduction approach. If the Mach stem is assumed as a straight face, the theoretical results agree well with the numerical results (Figs. 10a-10d).

\section{B. Effects of Sweep Angle and Wedge Angle}

Numerical and analytical results of corner flows with variation in sweep angle are shown in Figs. 11 and 12, where the flow conditions were selected as $M_{0}=5, \nu=\overline{90} \mathrm{deg}, \quad \theta=10 \mathrm{deg}$, and $\lambda=2 \sim 15 \mathrm{deg}$. The theoretical solutions to flowfield parameters with variations in wedge angle are presented in Fig. 13, where $M_{0}=5, \nu=90 \mathrm{deg}, \theta=4 \sim 35 \mathrm{deg}$, and $\lambda=0 \mathrm{deg}$. Shockpolar analysis and numerical simulations show that the shock interac- tion types in the aforementioned conditions are Mach interactions (Figs. 11 and 14).

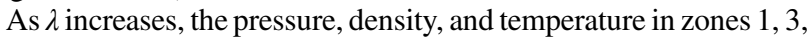
and 5 and the strength of the Mach stem decrease slightly, whereas the total pressure recovery coefficient slowly increases (Fig. 12a-12e). The total pressure recovery coefficients behind the incident waves and behind the reflected waves are almost identical (Fig. 12d)

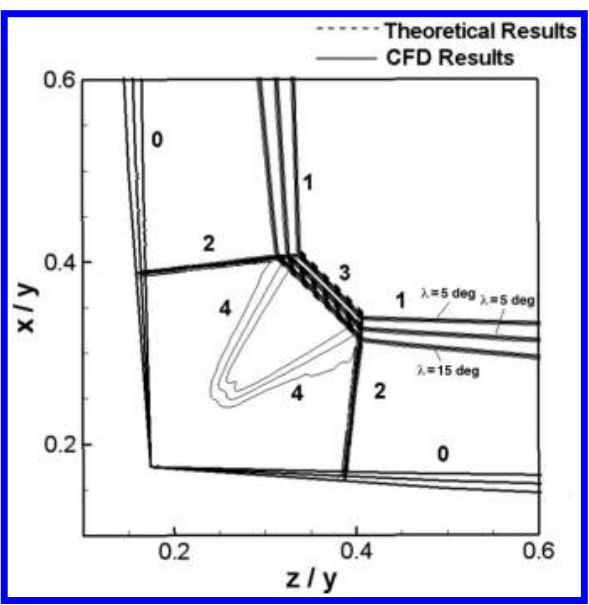

Fig. 11 Corner flow patterns of MI on cross section with $M_{0}=5$, $\nu=90 \mathrm{deg}, \theta=10 \mathrm{deg}$, SMI at $\lambda=5,10,15 \mathrm{deg} ; 0$ : wall, 1 : incident waves, 2: reflected waves, 3: Mach stem, 4: slip lines.

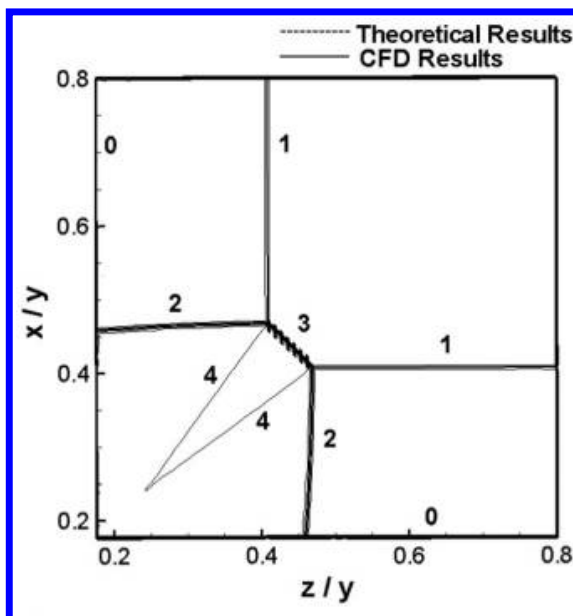

a)
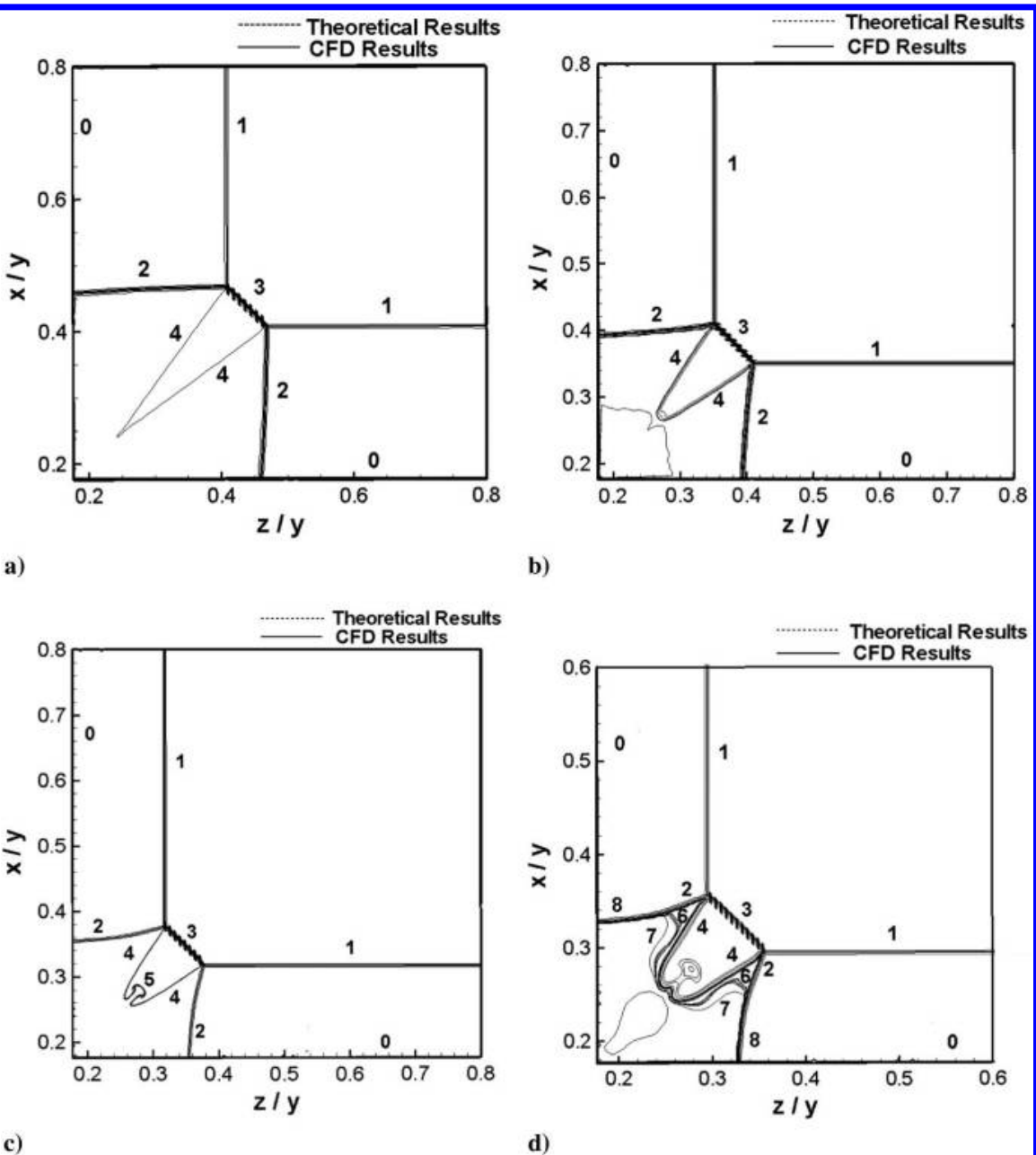

b)

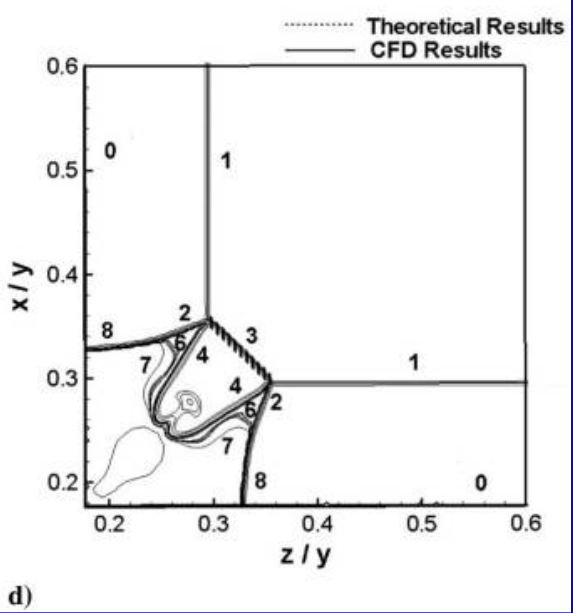

Fig. 10 Numerical wave configurations on cross section with $\nu=90 \mathrm{deg}, \lambda=0 \mathrm{deg}, \theta=10 \mathrm{deg}$ and theoretical results of Mach stem: a) SMI at $M_{0}=4$, b) SMI at $M_{0}=5$, c) TMI at $M_{0}=6$, and d) DMI at $M_{0}=7$. 
because the reflected wave is too weak to cause significant total pressure loss.

The numerical results in Fig. 11 show where wave configurations vary slightly as sweep angle increases. The boundary of the flowfield drops, the incident waves approach the axis, and the Mach stem nears the corner rib as the sweep angle increases; however, the reflected waves coincide fairly well with each other according to the numerical results (Fig. 11). It should be noted that, with the previous analysis, the effects of the sweep angle on the wave configuration and flowfield could be negligible.

For a large wedge angle (at $M_{0}=5, \nu=90 \mathrm{deg}$, and $\lambda=0 \mathrm{deg}$ ), shock waves over the wedges detach from the wedge; cases such as this are not considered here. Compared to variations in sweep angle, the impact of varying wedge angle on flowfield parameters and wave configuration is quite considerable (Figs. 13 and 14). As shown in the
Fig. 13, the pressure, density, temperature, and Mach stem strength in zones 1,3 , and 5 increase sharply as wedge angle increases, whereas the total pressure recovery coefficient decreases with the increase of wedge angle. At a 25 deg wedge angle, the pressure in zone 5 behind the Mach stem is about 14.2 times as much as that in zone 1, the temperature is about 4.2 times as much as that in zone 1 , and the total pressure behind the Mach stem is only about $20 \%$ that of the incoming flow (Fig. 13).

Numerical simulations show that wave configurations grow more complicated with larger wedge angle. The larger the wedge angle is, the narrower the distance between the wall and the waves is, influencing the corner flow on the waves. Under conditions where $M_{0}=5, \nu=90 \mathrm{deg}, \lambda=0 \mathrm{deg}$, and $\theta=10 \mathrm{deg}$, the shock interaction configuration is SMI (see Fig. $10 \mathrm{~b}$ ). Once the wedge angle is increased to $\theta=15 \mathrm{deg}$ or $\theta \overline{=2} 0 \mathrm{deg}$, the shock

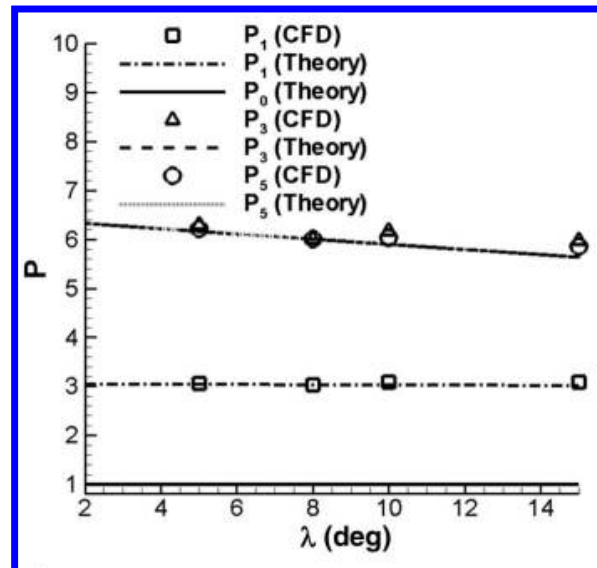

a)

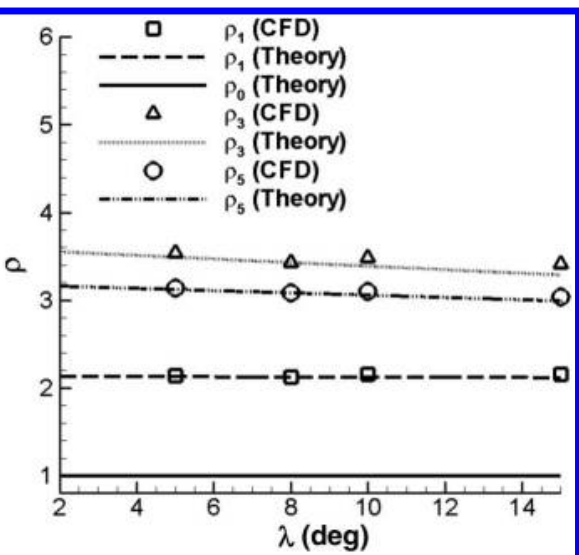

b)
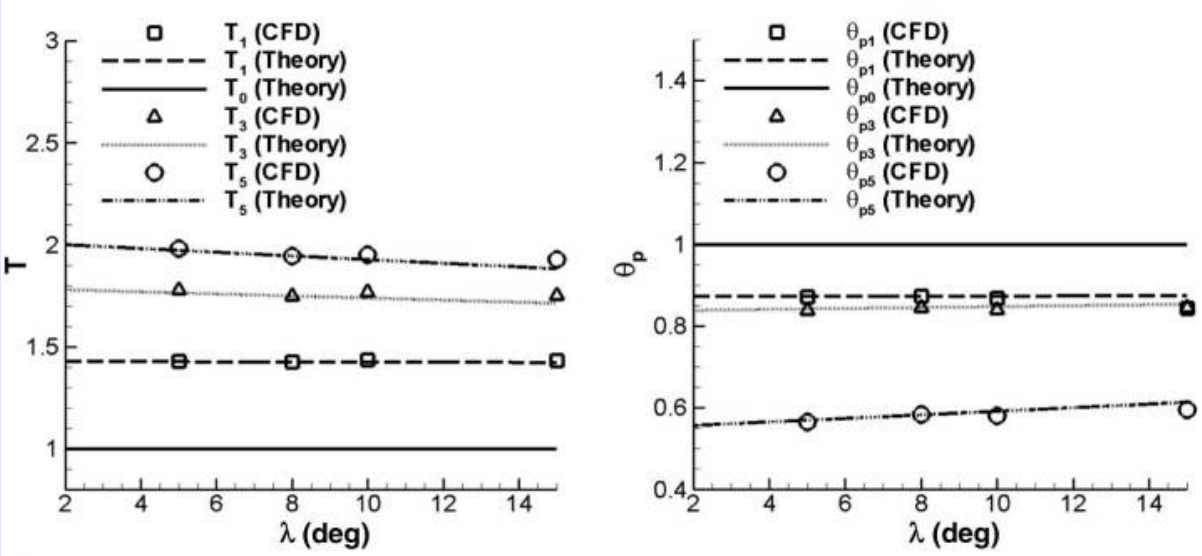

d)

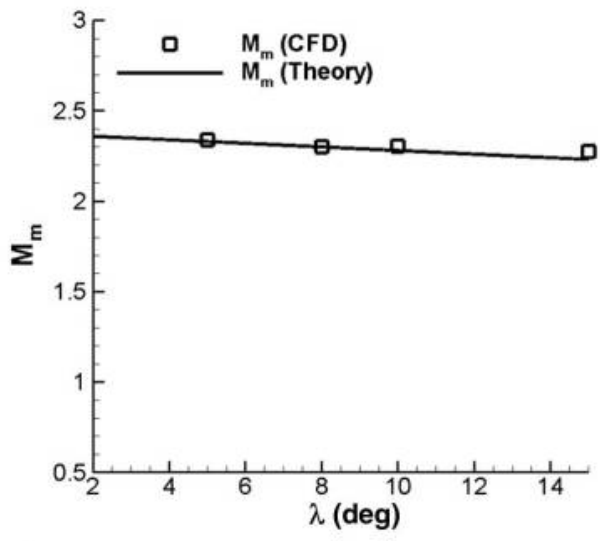

e)

Fig. 12 Results of flowfield parameter variations with sweep angle: a) pressure, b) density, c) temperature, d) total pressure recovery coefficient, and e) Mach stem strength. 

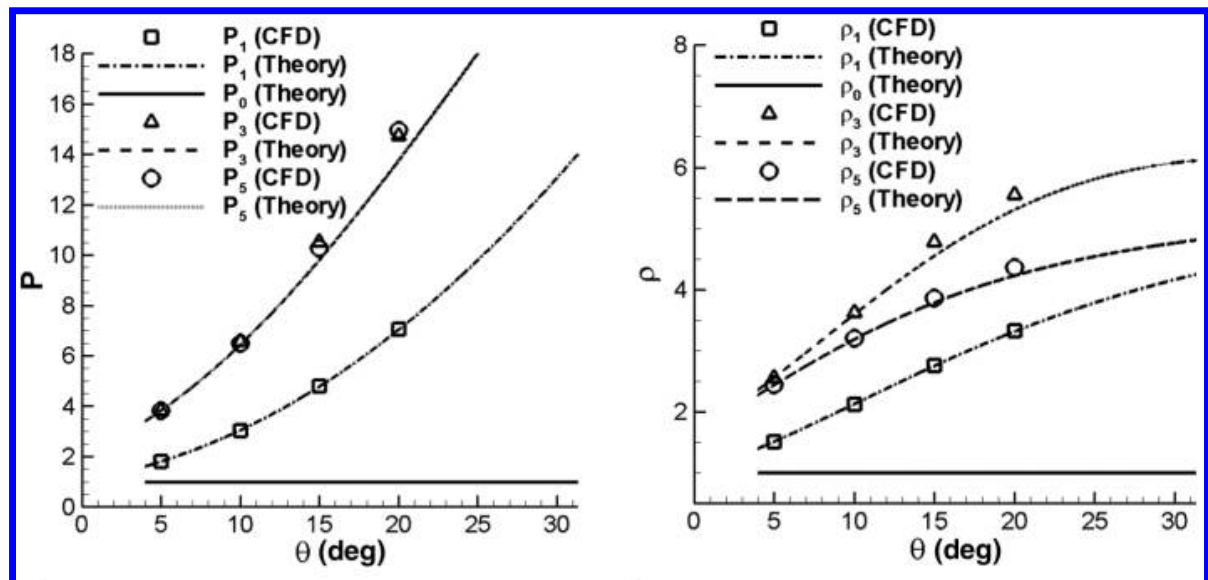

a)

b)
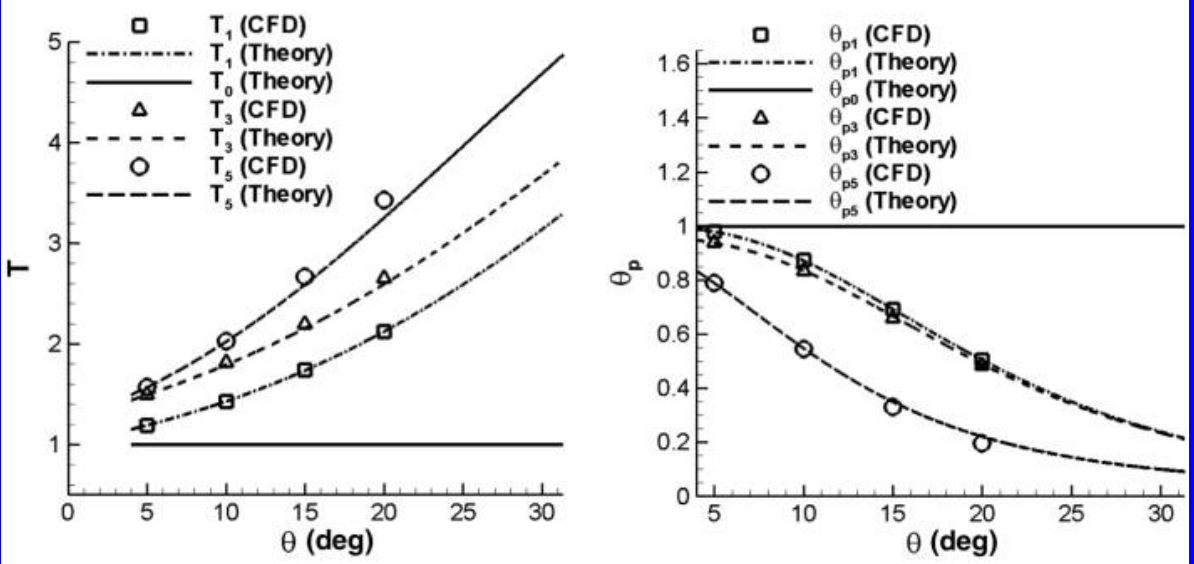

c)

d)

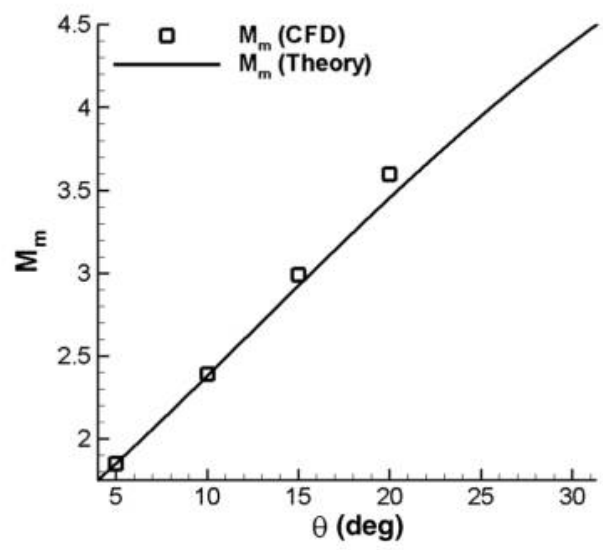

e)

Fig. 13 Flowfield parameter variations with wedge angle: a) pressure, b) density, c) temperature, d) total pressure recovery coefficient, and e) Mach stem strength.

interaction configuration is a DMI (Fig. 14a and 14b). If the wedge angle is increased further, the interactions are $\overline{\mathrm{DMI}}$ or multishock Mach interactions.

\section{Conclusions}

This study compared numerical simulations to an analytical solution to the problem of 3-D SSI over 3-D symmetric intersecting wedges. First, a novel method of spatial dimension reduction and the traditional numerical method were introduced; after working through the analytical method, results confirmed that the wave configuration can be effectively determined in agreement with the numerical results. Flowfield parameters were then obtained using the proposed approach, followed by both analytical and numerical analysis of the impact of key parameters on the flowfield and wave configurations. The most notable conclusions of this study can be summarized as follows.

1) 3-D wave configurations can be determined using the spatial dimension reduction approach, including both RI and MI. The RI flowfield near the reflection point and the MI in the vicinity of the triple point can be successfully obtained analytically.

2) For the MI configuration, the density, pressure, and temperature behind shock waves, as well as the Mach stem number, increase monotonously as Mach number increases, decrease slowly as sweep angle increases, and increase dramatically as wedge angle increases. The total pressure recovery coefficient decreases sharply as Mach number increases, increases slowly as sweep angle increases, and drops quickly as wedge angle increases. The effects of wedge angle 

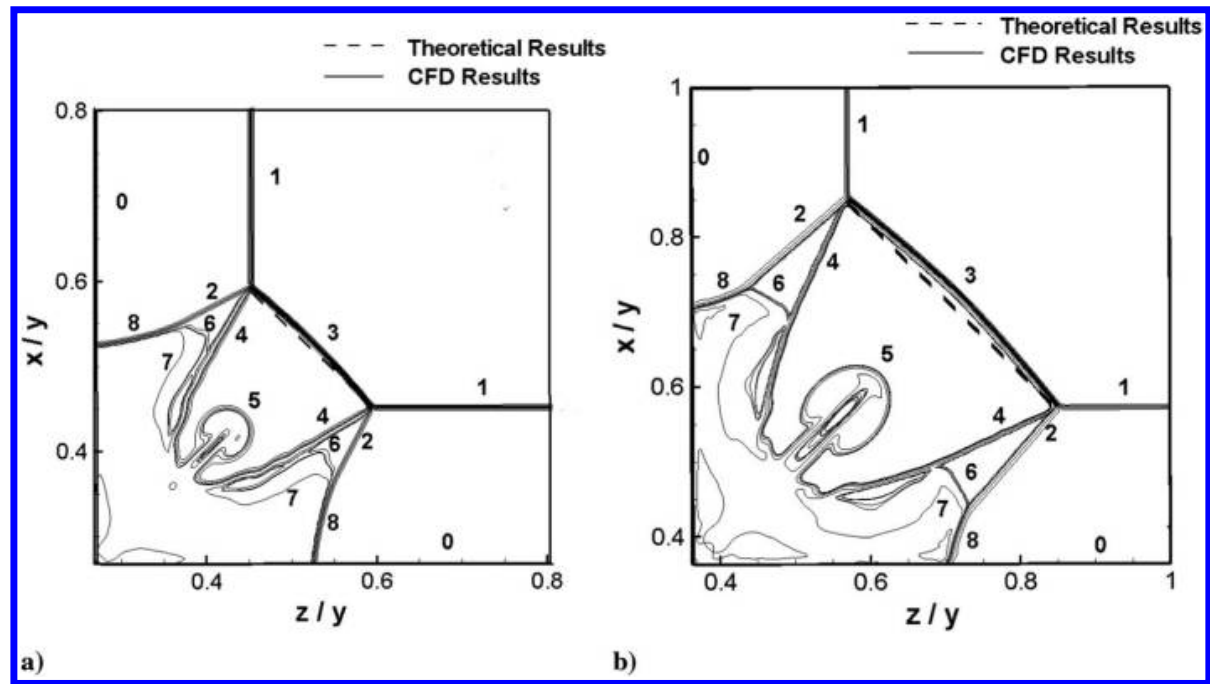

Fig. 14 Numerical wave configurations on cross section with $M_{0}=5, \nu=90 \mathrm{deg}, \lambda=0 \mathrm{deg}$ : a) DMI at $\theta=15 \mathrm{deg}$, and b) DMI at $\theta=20 \mathrm{deg}$.

on flowfield parameters are more pronounced than the effects of sweep angle.

3) Numerical simulations results show that larger Mach number and wedge angle lead to more complicated wave configurations. When Mach number and wedge angle are sufficiently large, the interaction type can be either double or multishock interaction. The influence of sweep angle on wave configuration is negligible.

4) As inflow Mach number, sweep angle, and wedge angle vary, 3-D steady shock interactions exhibit complex shock interaction configurations such as RI, SMI, and DMI. All the wave configurations in a 3-D steady SSI have corresponding wave configurations observed in the 2-D pseudosteady flows, such as RR, SMR, TMR, and DMR.

\section{Acknowledgments}

The project was supported by the National Natural Science Foundation of China (grants 11372333 and 90916028). We thank Luo and $\mathrm{Hu}$ for the help with the computing server.

\section{References}

[1] Mach, E., "Über den Verlauf von Funkenwellen in der Ebene und im Raume," Sitzugsbr Akad Wiss Wien, Vol. 54, 1878, pp. 819-838.

[2] von Neumann, J., "Refraction, Interaction and Reflection of Shock Waves," U.S. Navy, Bureau of Ordnance, Rept. 203-45, Washington, D.C., 1943.

[3] von Neumann, J., "Oblique Reflection of Shock Waves," Collected Work of John Von Neumann, Pergamon Press, Oxford, Vol. 145, No. 1, Dec. 1996, pp. 44-46.

[4] Kawamura, R., and Saito, H., "Reflection of Shock Waves-1 PseudoStationary Case," Journal of the Physical Society of Japan, Vol. 11, No. 5, 1956, pp. 584-592. doi:10.1143/JPSJ.11.584

[5] Ben-Dor, G., Shock Wave Reflection Phenomena, 2nd ed., edited by Davison, L., and Horie, Y., Springer-Verlag, Berlin, 2007, pp. 134-193.

[6] Ben-Dor, G., and Kawamura, R., "The Phenomena of Shock Wave Reflection-A Review of Unsolved Problems and Future Research Needs," Shock Waves, Vol. 2, No. 4, 1992, pp. 211-223. doi:10.1007/BF01414757

[7] Smith, L. G., "Photographic Investigation of the Reflection of Plane Shocks in Air," Physical Review, Vol. 69, No. 11-1, 1946, pp. 678-678.

[8] Ben-Dor, G., "Relation Between First and Second Triple-Point Trajectory Angles in Double Mach Reflection," AIAA Journal, Vol. 19, No. 4, 1981, pp. 531-533. doi: $10.2514 / 3.7791$

[9] Lee, J. H., and Glass, I. I., "Pseudo-Stationary Oblique-Shock-Wave Reflections in Frozen and Equilibrium Air," Progress in Aerospace
Sciences, Vol. 21, 1984, pp. 33-80. doi:10.1016/0376-0421(84)90003-4

[10] Charwat, A. F., and Redekeopp, L. G., "Supersonic Interference Flow Along the Corner of Intersecting Wedges," AIAA Journal, Vol. 5, No. 3, 1967, pp. 480-488. doi: $10.2514 / 3.4004$

[11] Watson, W., and Weinstein, L. M., "A Study of Hypersonic Corner Flow Interactions," AIAA Journal, Vol. 9, No. 7, 1971, pp. 1280-1286. doi: $10.2514 / 3.49937$

[12] Marconi, F., "Supersonic, Inviscid, Conical Corner Flowfields," AIAA Journal, Vol. 18, No. 1, 1980, pp. 78-84. doi: $10.2514 / 3.50733$

[13] Goonko, Y., Kudryavtsev, A., and Chpoun, A., "3D Interaction of Shock Waves in Corner Flow," Proceedings of the 24th International Symposium on Shock Waves, edited by Jiang, Z. L., Springer, Berlin, 2004, pp. 437-442.

[14] Goonko, Y., Kudryavtsev, A., and Rakhimov, R., "Supersonic Inviscid Corner Flows with Regular and Irregular Shock Interaction," Fluid Dynamics, Vol. 39, No. 2, 2004, pp. 304-318. doi:10.1023/B:FLUI.0000030314.27337.6c

[15] Skews, B. W., Mills, J. G., Quinn, P., Menon, N., and Mohan, J. A., "Supersonic Corner Flow with Fillets, Camber, Sweep and Dihedral," 25th International Symposium on Shock Waves, Bangalore, India, 2005, pp. 83-88.

[16] Naidoo, P., "Supersonic and Transonic Viscous Corner Flows," Ph.D. Thesis, Univ. of the Witwatersrand, Johannesburg, 2011.

-117] Naidoo, P., and Skews, B. W., "Supersonic Viscous Corner Flows," Proceedings of the Institution of Mechanical Engineers, Part G: Journal of Aerospace Engineering, Vol. 226, No. 8, 2011, pp. 950-965. doi:10.1177/0954410011416709

[18] Yang, Y., Wang, C., and Jiang, Z. L., "Analytical and Numerical Investigations of the Reflection of Asymmetric Nonstationary Shock Waves," Shock Waves, Vol. 22, No. 5, 2012, pp. 435-449. doi:10.1007/s00193-012-0392-9

[19] Xie, P., Han, Z. Y., and Takayama, K., "A Study of the Interaction Between Two Triple Points," Shock Waves, Vol. 14, No. 1, 2005, pp. 29-36. doi:10.1007/s00193-005-0245-x

[20] Milton, B. E., "Mach Reflection Using Ray-Shock Theory," AIAA Journal, Vol. 13, No. 11, 1975, pp. 1531-1533. doi: $10.2514 / 3.60566$

[21] West, J. E., and Korkegi, R. H., "Supersonic Interaction in the Corner of Intersecting Wedges at High Reynolds Numbers," AIAA Journal, Vol. 10, No. 5, 1972, pp. 652-656. doi: $10.2514 / 3.50171$ 